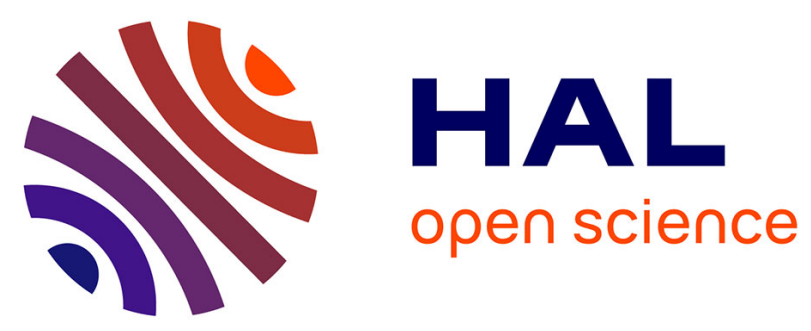

\title{
In situ measurements of interfacial contact pressure during impact hammer tests
}

Benjamin Seeger, Pauline Butaud, Volkan Baloglu, Fei Du, Matthew Brake, Christoph Schwingshakl

\section{- To cite this version:}

Benjamin Seeger, Pauline Butaud, Volkan Baloglu, Fei Du, Matthew Brake, et al.. In situ measurements of interfacial contact pressure during impact hammer tests. Conference and Exposition on Structural Dynamics, Feb 2018, Orlando, United States. 10.1007/978-3-319-74280-9_24 . hal02130074

\section{HAL Id: hal-02130074 \\ https://hal.science/hal-02130074}

Submitted on 15 May 2019

HAL is a multi-disciplinary open access archive for the deposit and dissemination of scientific research documents, whether they are published or not. The documents may come from teaching and research institutions in France or abroad, or from public or private research centers.
L'archive ouverte pluridisciplinaire HAL, est destinée au dépôt et à la diffusion de documents scientifiques de niveau recherche, publiés ou non, émanant des établissements d'enseignement et de recherche français ou étrangers, des laboratoires publics ou privés. 


\title{
In Situ Measurements of Interfacial Contact Pressure During Impact Hammer Tests
}

\author{
B. Seeger ${ }^{1}$, P. Butaud ${ }^{2}$, M.V. Baloglu ${ }^{3}$, F. Du ${ }^{4}$, M. R. W. Brake ${ }^{5}$, C. W. Schwingshackl ${ }^{6}$ \\ ${ }^{1}$ Institute of Aircraft Propulsion Systems, University of Stuttgart \\ Pfaffenwaldring 6, 70569 Stuttgart, Germany \\ ${ }^{2}$ Univ. Bourgogne Franche-Comté, FEMTO-ST Institute, CNRS/UFC/ENSMM/UTBM \\ Department of Applied Mechanics, 15B Avenue des Montboucons, 25030 Besançon, France \\ ${ }^{3}$ Chair of Applied Mechanics, Friedrich-Alexander University Erlangen-Nürnberg \\ Egerlandstr. 5, 91058 Erlangen, Germany \\ ${ }^{4}$ School of Astronautics, Northwestern Polytechnical University \\ No. 127 West Youyi Road, Xi'an, PR China \\ ${ }^{5}$ Department of Mechanical Engineering, William Marsh Rice University \\ 6100 Main St., Houston, TX 77251-1892, USA \\ ${ }^{6}$ Department of Mechanical Engineering, Imperial College London \\ South Kensington Campus, London SW7 2AZ, United Kingdom
}

\begin{abstract}
Understanding the nonlinear dynamical contact interactions within joints is crucial for understanding and predicting the dynamics of assembled structures. In spite of this, most experimental investigations focused on the global vibration behavior, since the local interactions at the interface cannot be observed with standard techniques. In the present work, an advance contact pressure measurement system is used in a unique way to analyze, in situ, the interfacial contact pressures and the contact area inside a bolted lap joint connecting two beams (Brake-Reuß beam). An important feature of the measurement system is that it is designed for frequency ranges including the typical vibration frequency of the Brake-Reuß beam's first eigenmode, and thus permits measurement under dynamic excitation. The dynamics of the contact pressures were investigated with different bolt torque levels and with different excitation levels. The experiments found that significant variations of the contact state occurred and that the contact pressure measurement system could adequately resolve this effect. The influence of the measurement system itself on the global vibration response of the Brake-Reuß beam was shown to be tolerable.
\end{abstract}

keywords: assembly, lapjoint, nonlinear, experimental, pressure sensor.

\section{Introduction}

Bolted joints are indispensable in most machines and mechanical devices. The interface characteristics of bolted joints, such as contact area and contact pressure distribution, greatly affect the dynamic characteristics of machines. Therefore, understanding the dynamical contact interactions that lead to a nonlinear dynamic response of a bolted lap joint is of great importance when it comes to the correct prediction of the dynamics of an assembled structure. However, it is very difficult to observe such behavior experimentally.

Until now, there are several techniques for contact area and contact pressure distribution measurements. Most of the actual techniques are static measurement methods, for example engineer's blue [1], optics $[2,3]$, ultrasound $[4,5,6,7,8]$ and pressure film measurements $[9,10,11,12]$. The widely used engineer's blue technique is convenient for contact area measurements, however it is not very accurate and cannot be used for contact pressure measurement. The optical technique is accurate but a large microscope and a camera are necessary and one contact surface should be transparent. The ultrasonic technique is noninvasive and has almost exclusively been used for static contact pressure measurements of bolt joints [4, 5]. The authors are only aware of one work using ultrasound for dynamic contact pressure measurements [8]. Under dynamic loading, a focused ultrasonic transducer as used in this work is only able to scan finite points, i.e. not a whole contact interface at once (thus only four discrete spots at the interface were measured in [8]).

Recently, Tekscan Inc. developed a digital pressure film using piezoresistive strips, suited for measurements of the dynamic contact pressure distribution and contact area by inserting the pressure film into the contact interface. In addition, it has a number of advantages, such as real-time monitoring capability, re-usability of the sensor and a direct computer interface. The signal acquisition system is capable of a sampling frequency up to $730 \mathrm{~Hz}$. 
In this paper, that Tekscan pressure sensor is used to measure, in situ, the dynamic contact area and contact pressure internal to a jointed interface during a transient response due to hammer excitation. A standard Brake-Reuß beam, consistent of two beams connected via a three bolt lap joint, is studied. Since the frequency range of the sensor includes the typical vibration frequency of the Brake-Reuß beam's first eigenmode, this sensor seems to be a promising tool for research on the nonlinear dynamic response of bolted joints. This paper is organized as follows: in section 2, the experimental setup is described, the impact of the sensor sheet on the global response of the BRB is discussed in section 3 and the recorded real time measurements of the contact pressure and area in the interface are presented in section 4 and section 5 .

\section{Experimental setup}

\subsection{Impact test setup}

To study the evolution of the contact pressure in the interface of a jointed system, the benchmark system of the BrakeReuß Beam (BRB) is used [13]. The BRB structure consists of two stainless steel beams, each with a cross section of $25.4 \mathrm{~mm} \times 25.4 \mathrm{~mm}$, connected via a three bolt lap joint, as seen in Fig. 1 and Fig. 3(a). The material of both beams is 304 stainless steel alloy and three $50 \mathrm{~mm} \mathrm{M8} \mathrm{bolts} \mathrm{are} \mathrm{used.} \mathrm{Each} \mathrm{bolt} \mathrm{is} \mathrm{tightened} \mathrm{using} \mathrm{a} \mathrm{torque} \mathrm{wrench.} \mathrm{Four} \mathrm{different}$ torque levels $(5 \mathrm{Nm}, 10 \mathrm{Nm}, 15 \mathrm{Nm}$ and $20 \mathrm{Nm}$ ) are applied to study the influence of the torque on the dynamically changing contact conditions in the interface. In order to obtain a better repeatability regarding the initial interface conditions, the bolts are tightened following the procedure established in [14]: first, $70 \%$ of the final torque is applied to each bolt, the center bolt being tightened first before the outer two bolts. Then the final torque is applied following the same order.

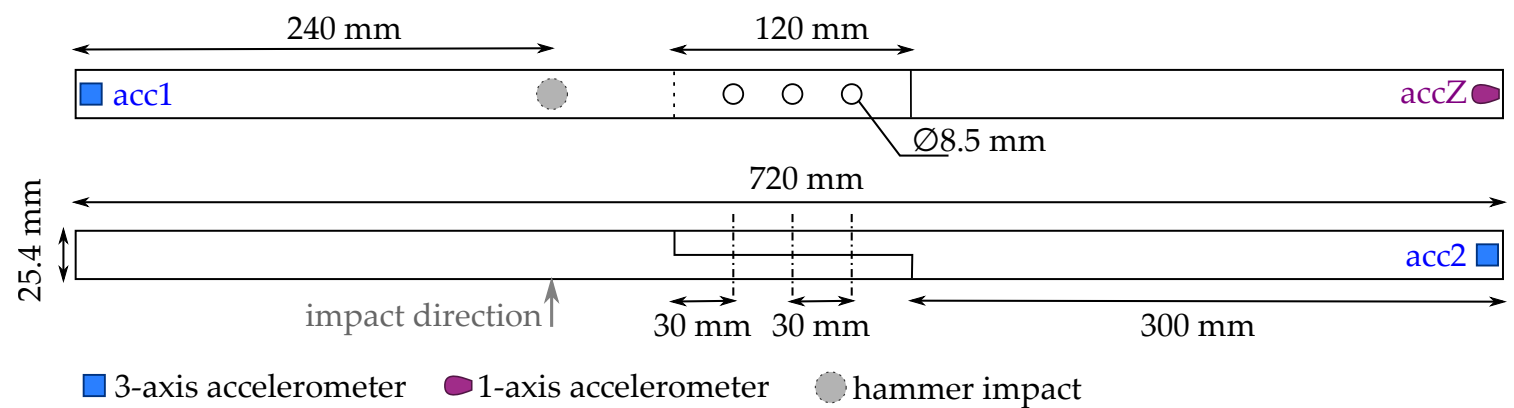

Figure 1: Profile and side view drawings of the Brake-Reuß Beam with the positions of the measurement and excitation systems.

The experimental setup, shown in Fig. 3(a), includes the beam, two bungee cords, two PCB 356A01 Triaxial ICP Accelerometers ("acc1" and "acc2"), a PCB 086D05 ICP Impact Hammer, a LMS 16 Channel Spectral Analyzer and a Tekscan shell sensor (described in section 2.2).

Following the setup described in $[15,16]$, the BRB beam is suspended $50 \mathrm{~mm}$ from each end with fishing lines connected to bungee cords in order to approximate free-free boundary conditions. The system is excited using an impact hammer at three different impact force levels: $60 \mathrm{~N}, 300 \mathrm{~N}$ and $600 \mathrm{~N}$. The point of impact is located $240 \mathrm{~mm}$ away of the end beam (grey circle on Fig. 1). For each excitation level and torque level, the measurements are repeated five times. The results presented in section 3 are the average of each set of five hits.

For the comparison of the BRB's response in terms of natural frequencies and damping with and without the sensor inserted in the interface, as presented in section 3.1, two three-axis accelerometers are used (blue squares in Fig. 1). For the vibration data associated with the interface pressure measurements presented in section 3.2 and section 5, respectively, only one PCB 352A21 mono-axial accelerometer ("accZ" in Fig. 1) is used. This is due to the fact that, in contrast to the mono-axial accelerometer, the three-axis accelerometers induced noise in the sensor sheet of the Tekscan system (see section 2.2). It is most likely that the higher power demand of the three-axis accelerometers (compared to the mono-axial one), in combination with the beam being made of steel and with the blinding of the Tekscan sensor sheet being insufficient in the interface pressure measurements setup detailed in the subsequent section, caused electrical interferences that compromised the sensor sheet. 


\subsection{Interface pressure measurements setup}

The thin-film based Tekscan system consists of a matrix of semi-conductive ink coating that creates a pressure dependent electrical resistance at intersection points called sensels or sensor pixels. The sensor matrix is encased in a polymeric material (specifically mylar). A schematic drawing of the Tekscan 5101 sensor sheet used in this study, extracted from its data sheet provided by Tekscan Inc. [17], is shown in Fig. 2. The sensor has an $109.22 \mathrm{~mm} \times 109.22 \mathrm{~mm}$ matrix $(\mathrm{MW} \times \mathrm{MH})$ consisting of 1936 sensels with a respective area of $6.4516 \mathrm{~mm}^{2}(\mathrm{CS} \times \mathrm{RS})$.

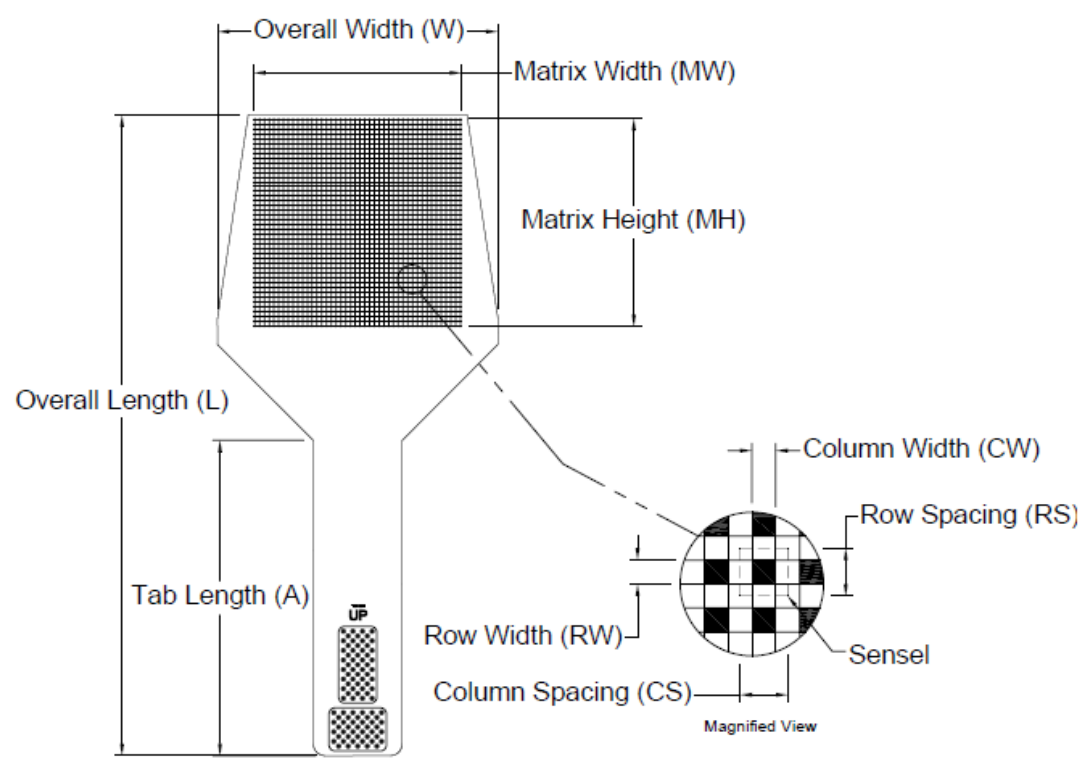

Figure 2: Tekscan Pressure Mapping Sensor 5101 [17]

At $3450 \mathrm{MPa}$, the pressure saturation of the sensor is reached. A sampling frequency of $650 \mathrm{~Hz}$, which is about four times the natural frequency of the BRB's first mode, was used to obtain the data presented in section 4 and section 5 .

The sensor sheet is placed inside the interface of the BRB and connected to the I-scan 7.60 software and the electrical power supply by a paddle, as shown in the Fig. 3(a). In order to be able to place the sensor sheet inside the lapjoint, three holes were required, made with a $\varnothing 8 \mathrm{~mm}$ punch. At the edges of these holes, the semi-conductive ink coating inside the sensor sheet is not covered anymore by the polymeric material. It was found that it severely compromises the sensor data if the ink coating and the metal bolts come into electrical contact. To prevent this, each bolt at the interface is taped to separate the edges of the sensor holes from the metal bolts. With the holes punched in the sensor, data is still able to be collected; however, portions of several rows and columns are lost. Fig. 3(b) illustrates the lost data (in red) when the sensor is placed at $45^{\circ}$ to the longitudinal axis of the beam. The pattern of the lost data results from the sensor's specific matrix architecture. The $45^{\circ}$ orientation is chosen to keep the maximum amount of information between the bolts and outside of the bolts, where the most interesting phenomena are assumed to happen.

(a)

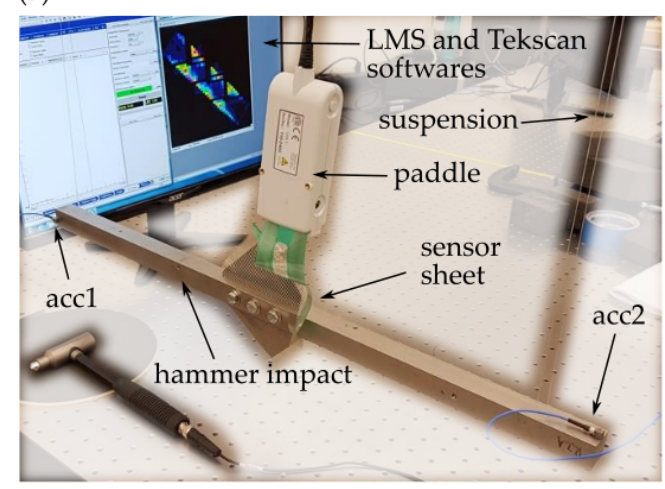

(b)

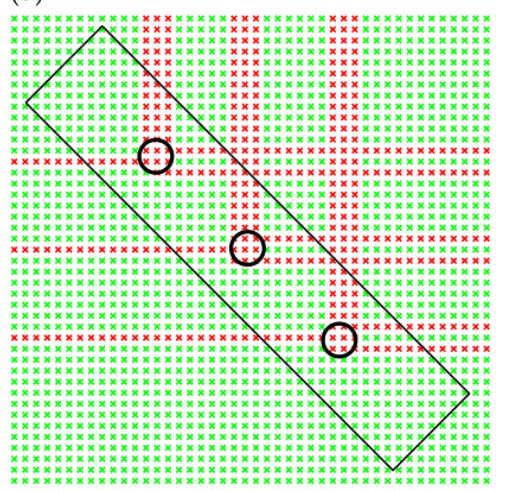

Figure 3: (a) Typical experimental setup of the BRB with the Tekscan sensor sheet. (b) Illustration of the sensels, with active sensels marked green, inactive sensels marked red and the contour of the beam interface drawn in black. 
The sensitivity of the Tekscan sensor can be adjust from one to 41, according to the pressure band of interest. The data obtained from the Tekscan sensor is dimensionless and given in "raw sum". The raw sum values of the sensor pixels represent the contact pressures relative to each other, i.e. not the absolute pressures at the pixels. A calibration using the area and applied force is required to obtain a scalar factor which converts the raw sum values into absolute pressure data. Since this paper is mainly concerned in monitoring the relative variation of pressure across the interface during a vibration cycle, and absolute values are not so much of relevance, the calibration process is not part of this paper.

\section{Influence of the interface measurement system on the global response}

In this section, the influence of inserting the sensor sheet in the interface of the Break-Reuß beam is studied. Inserting the polyester enveloped sensor introduces additional damping and flexibility to the interface, thus the quality of the measurements after changing the usual BRB interface has to be assessed. In the following, the BRB with the Tekscan sensor sheet placed in the interface is called BRBS (Brake-Reuß Beam with Sensor). Additionally, the variation of the nonlinear response with respect to changes of the bolt torque level and the impact force level for the BRBS are shown. Similar experiments with the focus on different interface geometries were performed in [14]. The here considered quantities, acquired from transient free decay time domain data with the help of the Hilbert transform outlined in [18] and [19], are the natural frequency and the damping ratio with respect to the amplitude. All values presented in the subsequent sections are the average of five hits, while every single measurement showed a very little deviation from its mean value. Fig. 4 shows a representative example for the deviation of the five hits to their calculated average, both for the BRB and the BRBS at a $5 \mathrm{Nm}$ bolt torque level and a high $(600 \mathrm{~N})$ impact force.
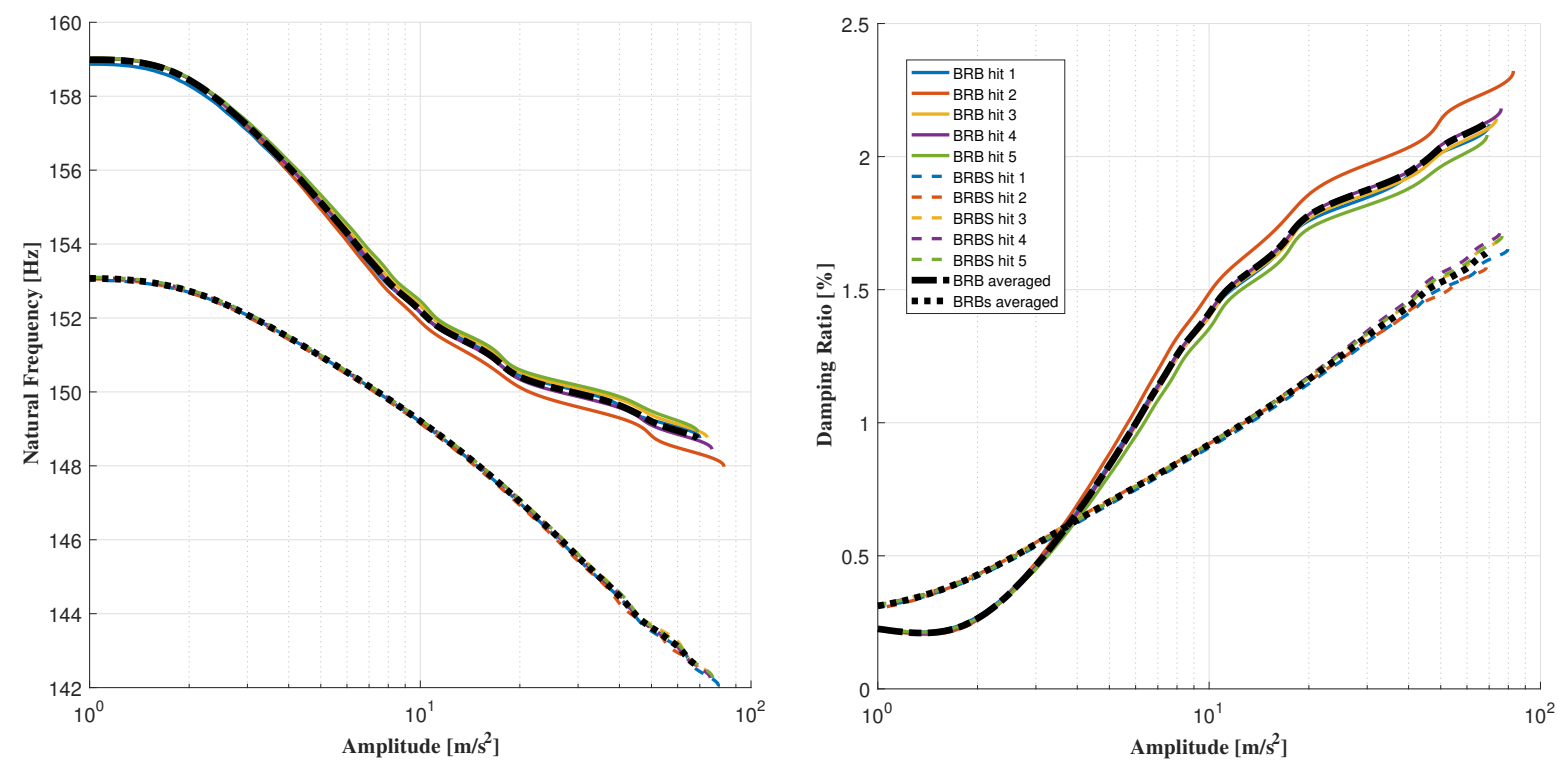

Figure 4: Average of five hits for a high $(600 \mathrm{~N})$ impact force with a $5 \mathrm{Nm}$ bolt torque, BRB and BRBS.

\subsection{Comparison between BRB and BRBS}

First, the natural frequencies and the damping of the BRB and the BRBS are compared for a constant bolt torque of $20 \mathrm{Nm}$ and three different impact force levels, starting at the low $(60 \mathrm{~N})$, exciting with the medium $(300 \mathrm{~N})$ and ending at the high $(600 \mathrm{~N})$ impact force level. The results, which are used to estimate the error caused by the sensor sheet, are shown in Fig. 5 .

The natural frequency, which seems to be independent from the excitation level in contrast to the amplitude, is shifted by an almost constant factor for every amplitude and is lower for the configuration with the sensor sheet. This expected softening behavior results from the polymeric casing material of the Tekscan sensor, which is weaker than the steel of the BRB. The damping ratio, by contrast, exhibits a slightly different behavior between the two systems. For the BRB, the measured damping ratios are not coincident for each excitation level (i.e. for higher excitation amplitudes, the damping ratio curves exhibit an increase above the previously measured levels). For the BRBS, however, the measured damping ratios for the medium and high excitation levels are coincident, that is, the measured curves overlap.

The ramifications of this result are that the dissipation mechanism between the two systems is slightly different. While for the BRB, the lack of coincidence in measurements could indicate evidence of modal coupling due to interfacial friction, 
the absence of these traits in the BRBS measurements indicate that more of the dissipation is coming from the polymeric film that constitutes the Tekscan sensor.
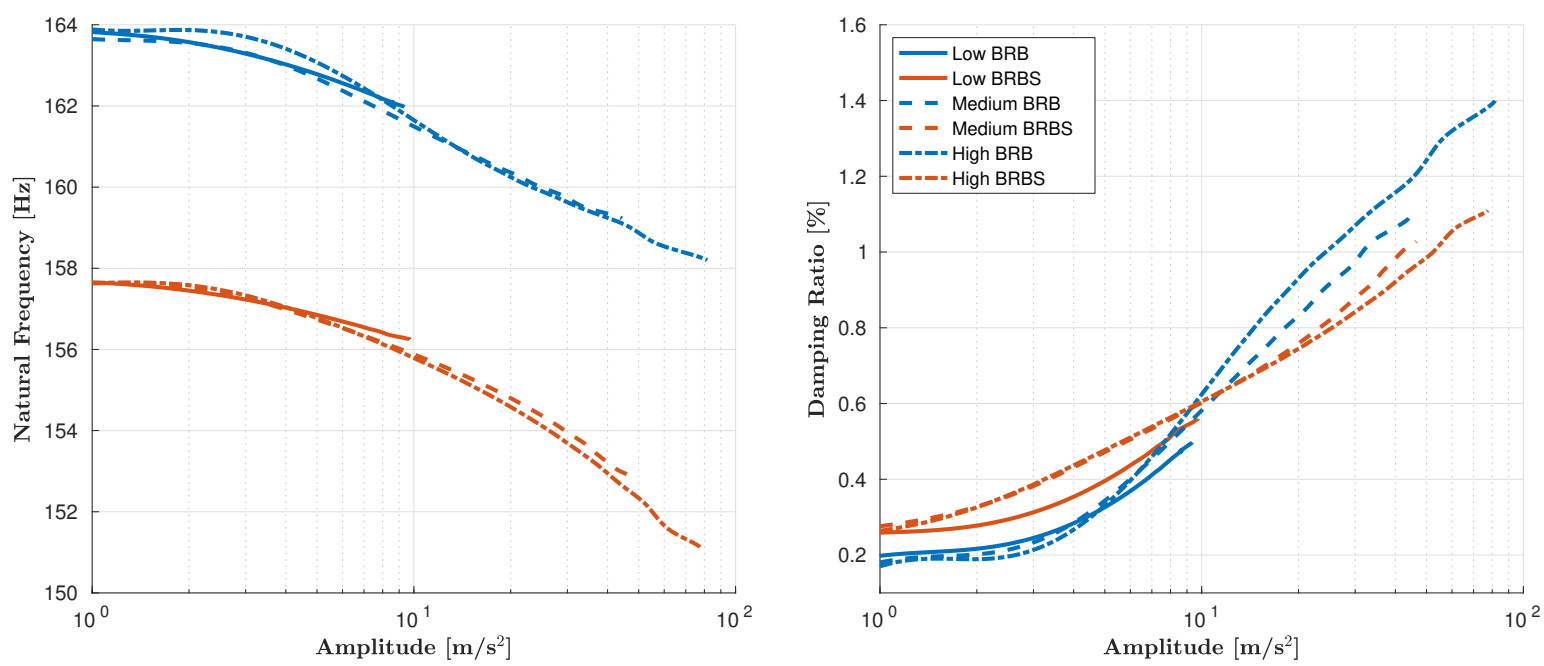

Figure 5: Comparison of the BRB and the BRBS for a low $(60 \mathrm{~N})$, medium $(300 \mathrm{~N})$ and high $(600 \mathrm{~N})$ impact force with a $20 \mathrm{Nm}$ bolt torque.

The damping ratio of the BRBS does not seem to be dependent on the amplitude as much as it is the case for the BRB. While for low amplitudes the damping ratio of the BRB is smaller than for the BRBS, the contrary is the case for higher amplitudes. One possible explanation is the polymeric material of the sensor sheet. At low amplitudes, the visco-elastic damping of the sensor sheet leads to higher damping of the BRBS compared to the BRB with its fully stuck interface. At high amplitudes, the interface of the BRB is sliding and dissipates more energy than the BRBS, which is still dominated by the visco-elastic damping. Nevertheless, these values stay in the same regime such that there is a justified hope to use the results of the BRBS for understanding the nonlinear dynamical contact interactions by keeping in mind that the natural frequency and the damping ratio are slightly affected in the above described way.

\subsection{Variation of bolt torque and impact force for the BRBS}

After the justification that the Break-Reuß beam with a sensor sheet placed at the interface can still produce representative results, four different bolt torque levels, namely $5 \mathrm{Nm}, 10 \mathrm{Nm}, 15 \mathrm{Nm}$ and $20 \mathrm{Nm}$, for the BRBS and a $600 \mathrm{~N}$ impact excitation are compared in Fig. 6.
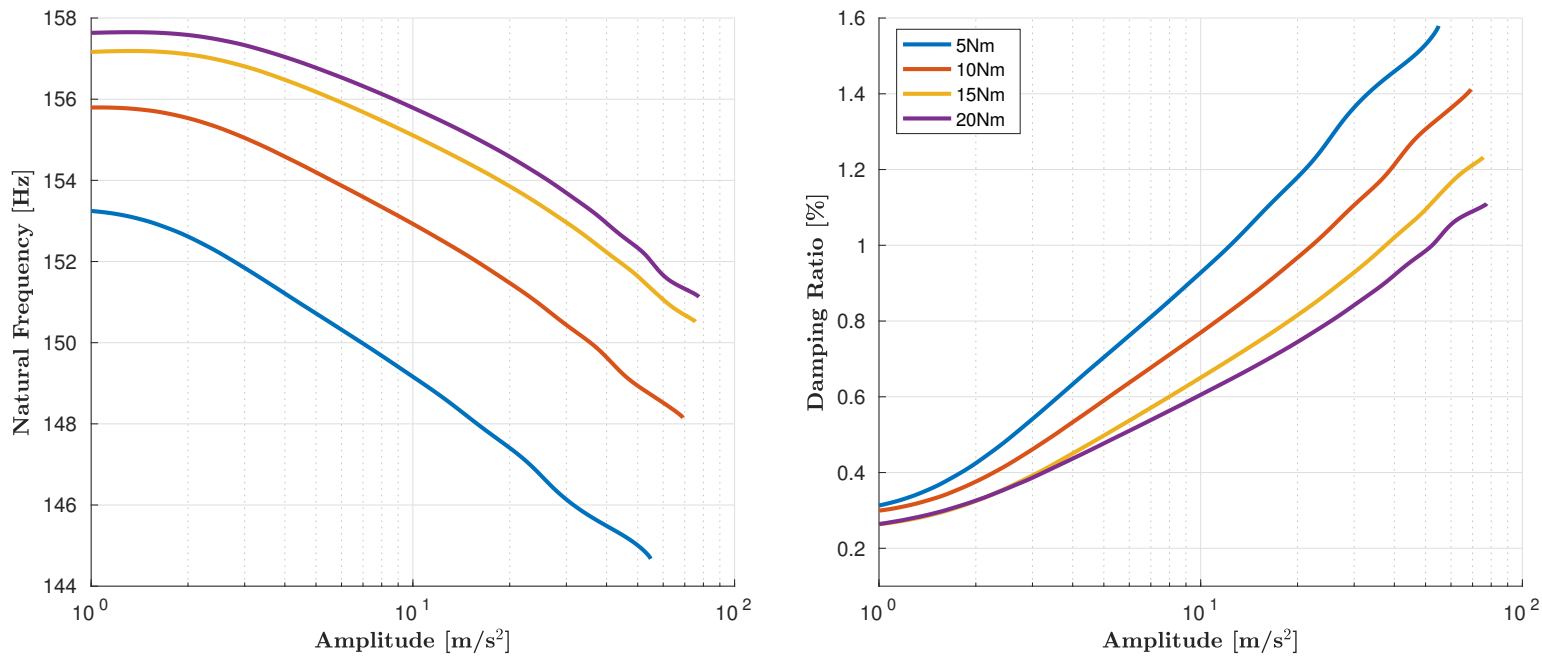

Figure 6: Comparison of different bolt torque levels for the BRBS and a high (600 N) impact force. 
It can be recognized that the applied torque has a noticeable influence on the natural frequency as well as the damping ratio. The natural frequencies of the four bolt torque levels are clearly distinguishable. The increasing preload leads to more stiffness in the interface and therefore to higher natural frequencies. The slope of the decrease of the natural frequencies at higher amplitudes is almost identical for all four bolt torque levels. Contrary to the natural frequency, the damping ratio becomes smaller with increasing bolt torque, since the increasing preload reduces the permissible relative motion in the interface and therefore the energy dissipation. Additionally, the slope of the damping ratio at higher amplitudes flattens with increasing bolt torque. The relative shift between the natural frequencies as well as between the damping ratios gets smaller the higher the preload gets. On the one hand, this indicates a saturation of the joint (the same effect could be observed for the nominal BRB in [15]). On the other hand, this also indicates that especially for the $5 \mathrm{Nm}$ case a strong softening of the system takes place and indicates additional frictional contact.

In the following, the two extreme cases at $5 \mathrm{Nm}$ and $20 \mathrm{Nm}$ bolt torque level are examined more closely. For these two bolt torque levels, the influence of the excitation force level on the natural frequency and damping ratio for the BRBS is further studied. Analogously to section 3.1, the BRBS is first excited at the low $(60 \mathrm{~N})$, then at the medium (300 N) and finally at the high $(600 \mathrm{~N})$ impact force level. The associated interface measurements of the first hit of each set of five hits are presented in section 5. Even though section 3.1 indicates that for $20 \mathrm{Nm}$ torque the force level does not heavily affect the calculated parameters, section 3.2 suggests that in combination with a lower torque this observation might not hold anymore. The results of this arrangement with three force levels and two torques are shown in Fig. 7.
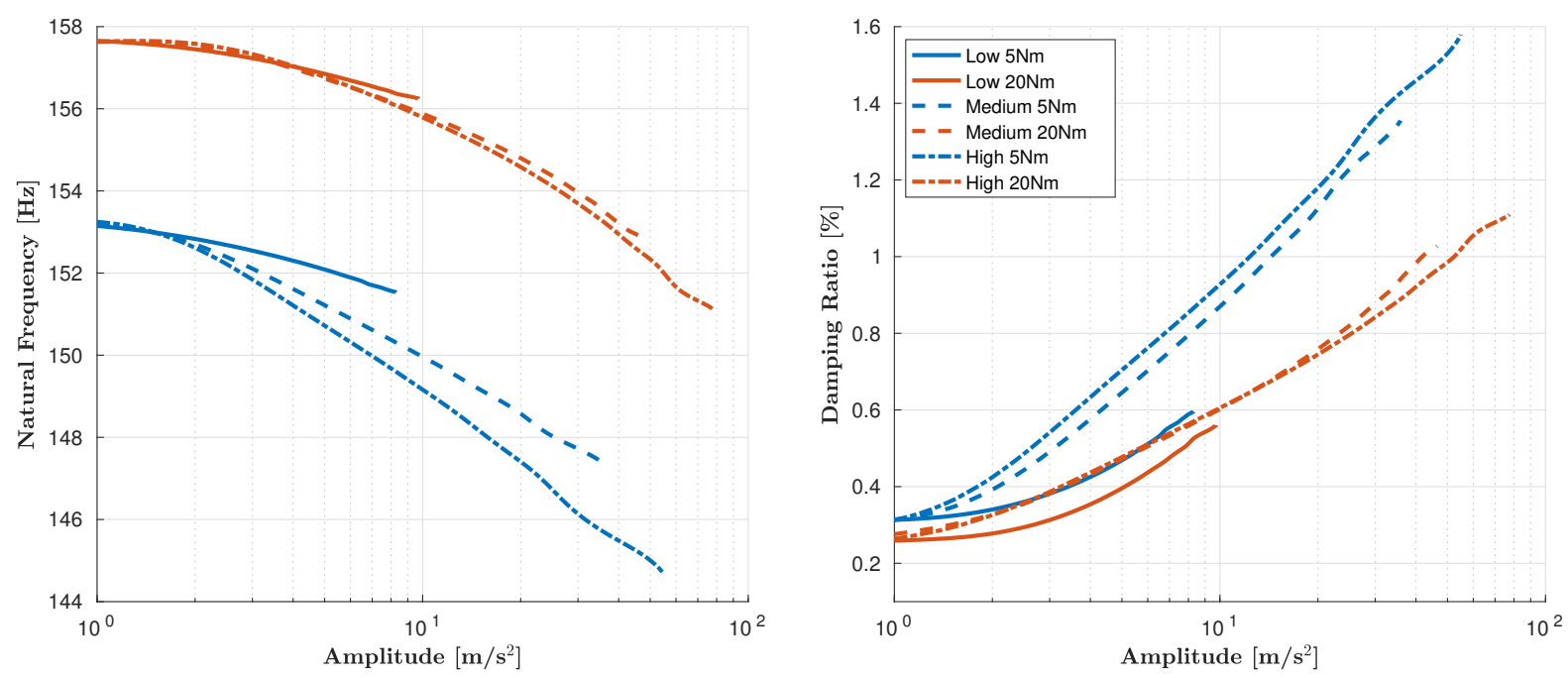

Figure 7: Comparison of the natural frequency and damping ratio for low (60 N), medium (300 N) and high (600 N) impact force with $5 \mathrm{Nm}$ and $20 \mathrm{Nm}$ bolt torque.

For the $5 \mathrm{Nm}$ bolt torque level, a significant difference between the frequency-vs-amplitude curves of the respective excitation level can be seen. The same difference is observable looking at the damping-vs-amplitude curves. This curve splitting depending on the excitation level strongly indicates several nonlinear phenomena. First, the energy dissipation and softening is due to the presence of microslip between the two surfaces (as opposed to macroslip, which would require relative motion between the two bodies). Second, that the curves for different amplitudes do not overlap is indicative of modal coupling. As more energy is put into the system, the nonlinearity of the interface excites multiple higher modes. Thus, as the excitation level is increased, more modes are contributing to the measured "mode 1" response. These results are further explored in section 5.

The results of section 3.1 and section 3.2 show that the sensor sheet in the interface induces a frequency shift, but the relative change of the BRB's natural frequencies with respect to the bolt torque level can still be assessed. The damping ratios of the BRBS stay in the same regime as the damping ratios of the nominal BRB. However, it is assumed that the dominating damping mechanism in case of the BRBS is different from the dominating damping mechanism of the BRB (c.f. section 3.1), thus one has to be cautious when comparing the damping ratios.

\section{Validation of the interface measurement system}

After investigating the influence of the sensor sheet on the global response, now the capabilities of the Tekscan system itself are assessed.

The static loading of the interface is done simply by tightening the beam's three bolts. Two different bolt torque levels 
are applied, namely $5 \mathrm{Nm}$ and $20 \mathrm{Nm}$. First, the pressure distribution inside the interface is measured with a Fujifilm pressure measurement film, then the Tekscan sensor sheet is used. The sensitivity chosen for the Tekscan sensor sheet (sensitivity $=6$, c.f. section 2.2) and the pressure range of the Fujifilm pressure measurement film (medium) are the same for both bolt torque levels. Fig. 8 shows the interface pressure distribution at $5 \mathrm{Nm}$, the pressure distribution at $20 \mathrm{Nm}$ is shown in Fig. 9. In the Tekscan sensor output, the contour of the beam interface is displayed in white.
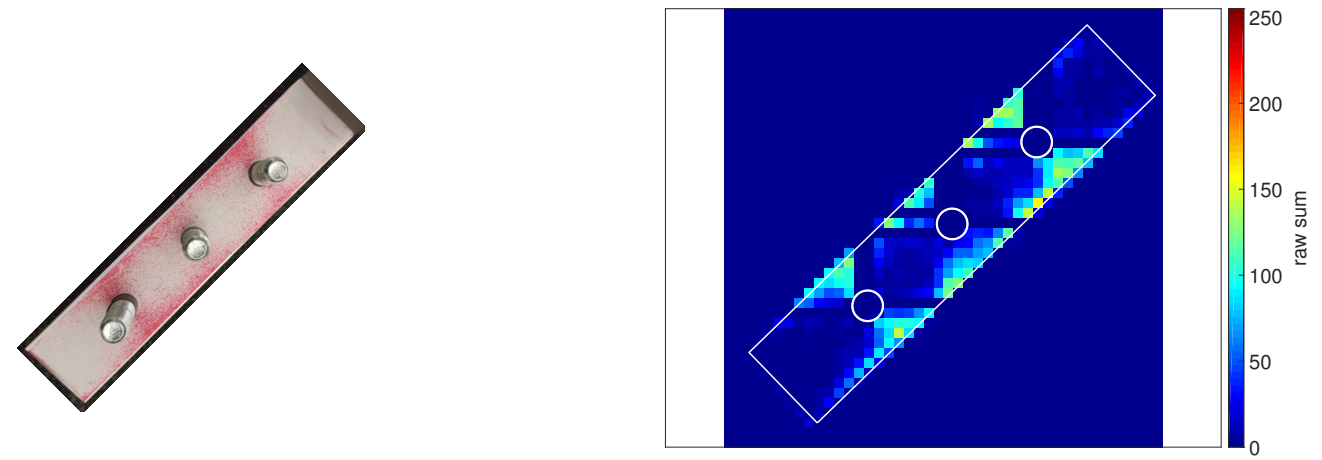

Figure 8: Pressure field at $5 \mathrm{Nm}$ with (left) Fujifilm and (right) Tekscan sensor.
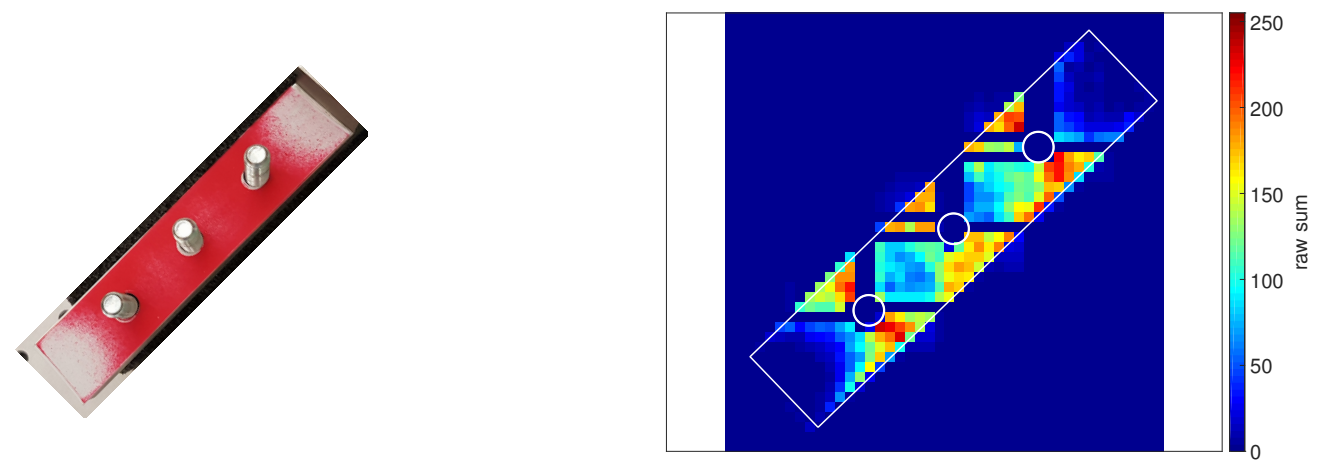

Figure 9: Pressure field at $20 \mathrm{Nm}$ with (left) Fujifilm and (right) Tekscan sensor.

The difference in distribution and magnitude of the interfacial contact pressure at $5 \mathrm{Nm}$ and $20 \mathrm{Nm}$ (shown in Fig. 9) is clearly visible in the output of the Tekscan sensor sheet. ${ }^{1}$ At $5 \mathrm{Nm}$ bolt torque level (Fig. 8), the contact pressure in the areas between the bolt holes and in the areas outside of the bolt holes towards the short edges of the interface is close to zero. The contact pressure is concentrated along the long edges of the interface, mainly in the vicinity of the bolt holes. At $20 \mathrm{Nm}$ bolt torque level (Fig. 9), a higher contact pressure is measured in the area between the bolt holes, while the contact pressure in the areas outside of the bolt holes towards the short edges of the interface is still close to zero. Analogously to the $5 \mathrm{Nm}$ case, most of the contact pressure is focused in the vicinity of the bolt holes and towards the long edges of the interface, but now the contact pressure is significantly higher. This distribution of the interfacial contact pressure (both, at $5 \mathrm{Nm}$ and $20 \mathrm{Nm}$ ) shows that the interface of the BRB used for the experiments is not flat, but exhibits a curvature orthogonal to the longitudinal axis of the interface. The results of the Tekscan sensor are consistent with the image on the Fujifilm pressure measurement film. Although some information is lost due to the necessary bolt holes (c.f. section 2.2), the pressure distribution between the bolt holes and outside the bolt holes is still well represented in the Tekscan setup.

In order to assess the capabilities of the Tekscan system with respect to dynamic loading, hammer impact tests were conducted with the sensor sheet placed inside the interface as shown in Fig. 3. As previously mentioned in section 2, the sampling rate of the Tekscan system was $650 \mathrm{~Hz}$. In the current section, the output of the sensor sheet under dynamic loading is only briefly illustrated, while the results of the dynamic interface measurements are presented and discussed in section 5 .

\footnotetext{
${ }^{1}$ Note that the static pressure film appears saturated, whereas the electronic pressure film shows clear gradients in the measured contact pressure. While these gradients may be evident in a digital analysis of the static pressure film, that information is inaccessible without a significant amount of work.
} 

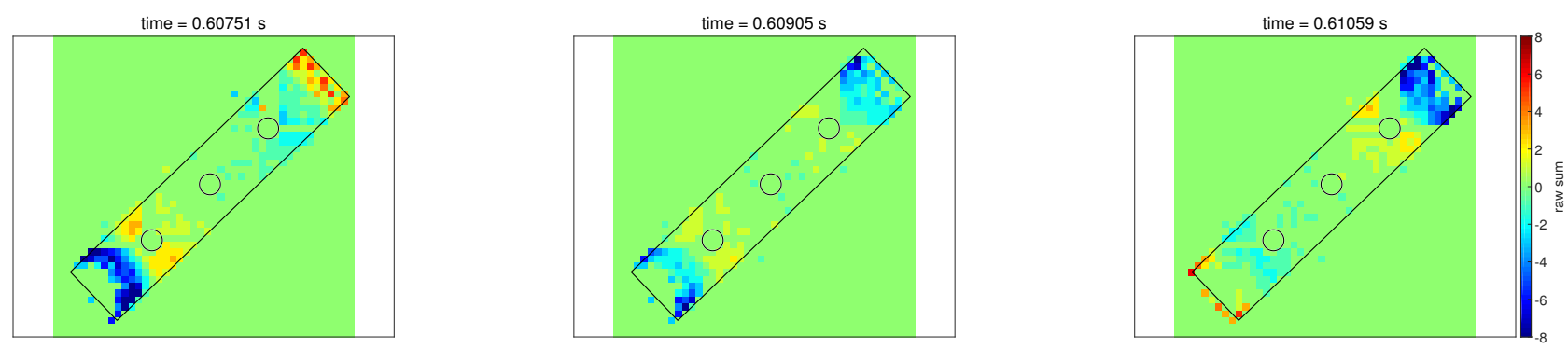

Figure 10: Evolution of the dynamic contact pressure at $20 \mathrm{Nm}$ bolt torque and medium excitation level.

In Fig. 10, three consecutive frames of Tekscan sensor sheet output at medium excitation level (300 N) and $20 \mathrm{Nm}$ bolt torque level are shown. The left frame was recorded $0.03076 \mathrm{~s}$ after the hammer impact at $t=0.57675 \mathrm{~s}$. This time shift (with respect to the hammer impact) of the frames shown in Fig. 10 is chosen in order to be able to illustrate the change of the dynamic contact pressure mainly by means of the first mode. ${ }^{2}$ For a better visibility of the dynamic portion of the contact pressure, the static pressure distribution as seen in Fig. 9(right) is subtracted from the original sensor sheet output data which consists of static and dynamic portions of the contact pressure.

Comparing the three frames in Fig. 10, one can clearly see the change of the pressure distribution in the area outside of the bolt holes towards the short edges of the interface. There, an unloading is followed by an increase in pressure. Additionally, one can observe a change of the contact pressure directly inside the pressure cones of the bolts. This demonstrates the Tekscan system's ability of capturing the evolution of the interfacial contact pressure under dynamic loading.

\section{Dynamic interface measurements}

In this section, the results of the interface measurements taken during the impact hammer tests associated with Fig. 7 are presented. While the results shown in Fig. 7 are the average of five hits in the respective configuration, the results of the interface measurements presented in this section are the results at the first hit of each set of five hits. Measurements were taken at $5 \mathrm{Nm}$ and $20 \mathrm{Nm}$ bolt torque level, each level first excited at the low $(60 \mathrm{~N})$, then at the medium $(300 \mathrm{~N})$ and finally at the high $(600 \mathrm{~N})$ impact force level.

In Fig. 11, the normalized contact area over time is shown for both bolt torque levels. The contact area is calculated by summing up the number of sensor pixels with a raw sum greater than zero for each measurement frame, yielding one scalar value per frame. The normalization is with respect to the contact area of a measurement frame prior to the hammer impact, i.e. the contact area under static loading. Comparing the left hand $(5 \mathrm{Nm})$ and right hand $(20 \mathrm{Nm})$ side of Fig. 11 , one can observe that especially during the first $0.1 \mathrm{~s}$ after the impact, the relative loss of contact area at medium and high excitation is significantly higher for the $5 \mathrm{Nm}$ bolt torque level than for $20 \mathrm{Nm}$. This corresponds to the frequency shift in Fig. 7, since the less pre-stressed interface at $5 \mathrm{Nm}$ allows more motion in normal direction than at $20 \mathrm{Nm}$, i.e. softening the system by additional separation.

All output signals of the normalized contact area in Fig. 11 show the same overall behavior: the signal drops after the hammer impact, followed by an oscillation back towards the initial value of one. It has to be noted that at in Fig. 11, the normalized contact area for the $20 \mathrm{Nm}$ case does not go back to one again. Considering the (positive) slope of the output signal towards the end, the most probable explanation is that the signal acquisition time is insufficient for the $20 \mathrm{Nm}$ case and the responses at the respective excitation levels have not fully decayed.

The relative change of the contact pressure is shown in Fig. 12. The normalized raw sum of each measurement frame is the sum of the raw sum values of all sensor pixels (c.f. section 2.2), normalized with respect to the sum of the raw sum values under static loading, analogously to the normalized contact area. The same measurement frame was used for the normalization of the raw sum and for the normalization of the corresponding contact area. Taking a look at Fig. 12, we see that the relative loss of the contact pressure under medium and high excitation is significantly higher for the $5 \mathrm{Nm}$ bolt torque level than for $20 \mathrm{Nm}$.

\footnotetext{
${ }^{2}$ Immediately after the hammer impact, the sensor sheet output consists of a superposition of the dynamic contact pressures generated by all participating modes in the frequency band excited by the impact (unfortunately, no low-pass filter could be applied on the output signal of the sensor sheet). Assuming a similar modal damping for all modes, the higher modes decay faster than the lower modes due to their higher vibration frequencies. Thus it seems reasonable that some time after the impact, the first mode is dominating the response and most of the dynamic contact pressure is generated by that first mode.
} 

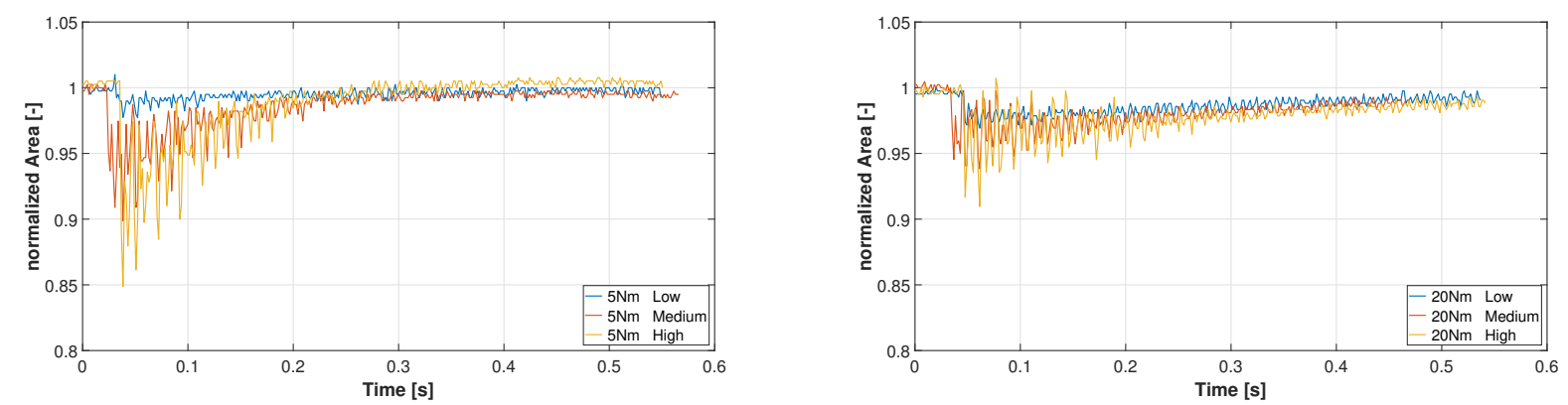

Figure 11: Normalized contact area over time at (left) $5 \mathrm{Nm}$ and (right) $20 \mathrm{Nm}$ bolt torque level for low, medium and high excitation.
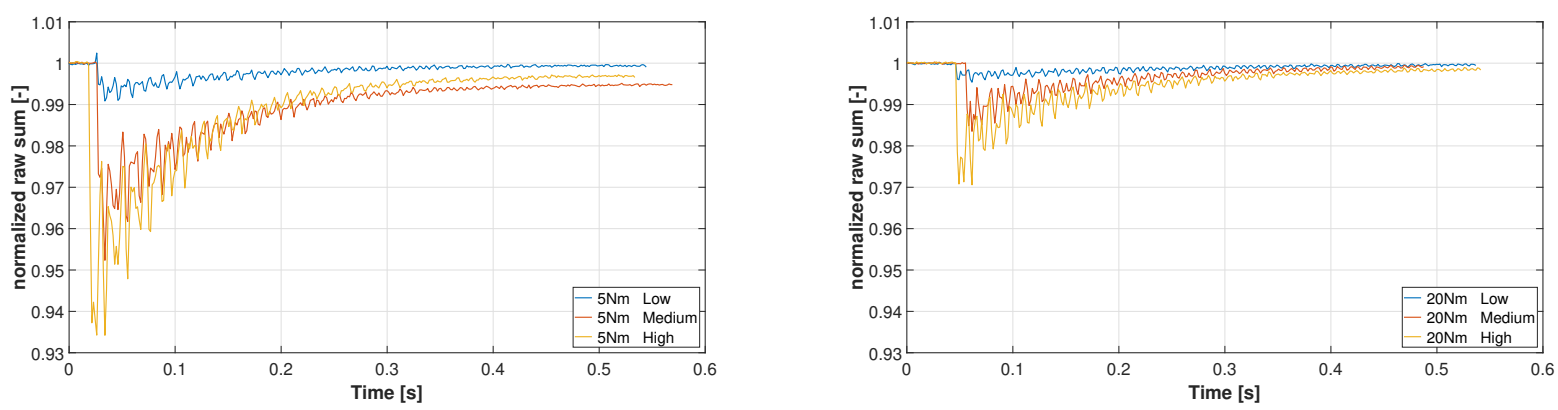

Figure 12: Normalized raw sum over time at (left) $5 \mathrm{Nm}$ and (right) $20 \mathrm{Nm}$ bolt torque level for low, medium and high excitation.

The output signals of the normalized raw sum values for the $20 \mathrm{Nm}$ case (right hand side Fig. 12) show the same behavior as the normalized contact areas: the signal drops after the hammer impact, followed by an oscillation back towards the initial value of one. Contrary to that, the normalized raw sum values for the three excitation levels with $5 \mathrm{Nm}$ bolt torque clearly exhibit a difference at the end of the respective measurements. At a low excitation level, the normalized raw sum equals one again. After the impact at the medium excitation, the normalized raw sum does not reach again the initial value of one (here, the slope of the output signal is zero towards the end, implying a fully decayed response). Keeping in mind the order of the excitation levels (low, medium, high), this strongly indicates a different contact state in the interface after the impact, i.e. that microslip occurred due to the impact. The same holds for the normalized raw sum before and after the impact at high excitation level. This observation corresponds to the splitting of the frequency-vs-amplitude curves at $5 \mathrm{Nm}$ bolt torque level in Fig. 7.

An interesting observation at both bolt torque levels is the simultaneous drop of the contact area (Fig. 11) and the corresponding contact pressure (Fig. 12) which, at a first glance, seems somewhat counter-intuitive. Under the assumption of a constant bolt load, one would expect the contact pressure to increase when the contact area gets smaller and vice versa. A possible explanation can be given by using the perspective that the bolts and the beams can be represented as two springs in parallel [20]. The representation of the bolts as tension springs and the beams as compression springs is illustrated in Fig. 13 .

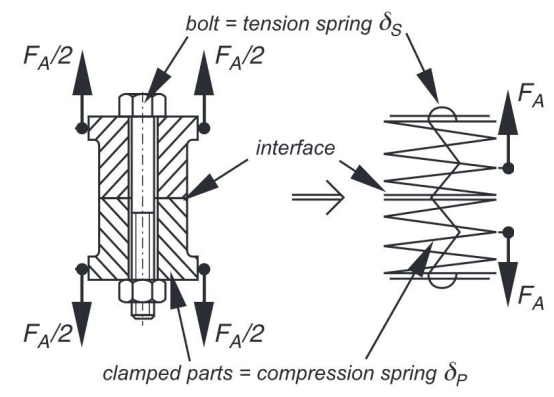

Figure 13: Conversion of a clamped joint into the spring model [20] 
In the static case, the bolt torque generates an axial force in the bolts and a corresponding reaction force in the beams. Because of the bolts' compliance $\delta_{S}$ and due to the axial force, the bolts elongate. The corresponding reaction force compresses the beams due to the beams' compliance $\delta_{P}$. This induces a tensile stress in the bolts and a compression stress in the beams, the latter giving us the static contact pressure distribution measured in the interface (e.g. Fig. 9). Now we consider a stress wave propagating through the lap joint due to the hammer impact. This stress wave induces an additional axial force $F_{A}$ acting on the clamped joint, as depicted in Fig. 13. Due to $F_{A}$ and because of the axial compliance $\delta_{S}$, the bolts additionally elongate, yielding a displacement $u_{a x}$ and an additional tensile stress in the bolts. Because both "springs" are in parallel, the axial compression of the beams is reduced by the same displacement $u_{a x}$. Now, by virtue of $\delta_{P}$, the axial force acting on the beams is reduced which in turn allows for additional separation and a reduction of the contact pressure in the interface at the same time. This redistribution of the axial forces and the corresponding tensile and compression stresses takes place as long as the stress wave inducing $F_{A}$ propagates through the lap joint. ${ }^{3}$

Therefore, the simultaneous drop of the contact area and the contact pressure in the interface could be explained with a temporary transfer of potential (i.e spring) energy from the beams to the bolts.

\section{Conclusions and perspectives}

In this work, a new approach for dynamically measuring contact pressures in a bolted assembly was explored. This initial study focused on the capability of the new technique, electronic pressure films, to capture the transient events associated with impact hammer testing. The experiments found that significant variations in contact pressure occurred as a stress wave propagated through the interface and that the electronic pressure film could adequately resolve this effect.

Additionally, the preliminary results from these experiments indicate that several commonly held assumptions for modeling jointed structures may be invalid: that contact pressure is constant under the frustrum of the bolt, that the system can be approximated to have no modal coupling, and that there is no dynamic impact internal to the interface. Instead, this research presents evidence to the contrary for each of these common assumptions. Future work must rigorously investigate these assumptions in order to develop a new approach for modeling jointed structures. In particular, by exciting the BRBS with a shaker at a frequency close to a natural frequency, the interfacial contact pressure interactions due to the mode belonging to that particular natural frequency could be isolated and investigated, thus elucidating new understanding to the physics of joint dynamics and the nature of the nonlinear coupling observed.

\section{Acknowledgments}

This research was conducted at the 2017 Nonlinear Dynamics of Coupled Structures and Interfaces Summer Research Program, sponsored by Rice University.

The authors would also like to thank the "Fond Interministeriel Unique" that funds the project CLIMA and the "BourseOréal UNESCO Pour les Femmes et la Science" encouraging women to pursue careers in science.

The authors would also like to thank the National Natural Science Foundation of China (Grant No.51705422).

\section{References}

[1] KL Woo and TR Thomas. Contact of rough surfaces: a review of experimental work. Wear, 58(2):331-340, 1980.

[2] A Ovcharenko, G Halperin, I Etsion, and M Varenberg. A novel test rig for in situ and real time optical measurement of the contact area evolution during pre-sliding of a spherical contact. Tribology Letters, 23(1):55-63, 2006.

[3] Masao Eguchi, Takashi Shibamiya, and Takashi Yamamoto. Measurement of real contact area and analysis of stick/slip region. Tribology International, 42(11):1781-1791, 2009.

[4] RS Dwyer-Joyce. The application of ultrasonic ndt techniques in tribology. Proceedings of the Institution of Mechanical Engineers, Part J: Journal of Engineering Tribology, 219(5):347-366, 2005.

[5] Massimiliano Pau and Antonio Baldi. Application of an ultrasonic technique to assess contact performance of bolted joints. Journal of Pressure Vessel Technology, 129(1):175-185, 2007.

[6] Fei Du, Jun Hong, and Ying Xu. An acoustic model for stiffness measurement of tribological interface using ultrasound. Tribology International, 73:70-77, 2014

[7] Fei Du, Baotong Li, Jie Zhang, Quan Min Zhu, and Jun Hong. Ultrasonic measurement of contact stiffness and pressure distribution on spindleholder taper interfaces. International Journal of Machine Tools and Manufacture, 97:18-28, 2015.

[8] M. B. Marshall, I. Zainal, and R. Lewis. Influence of the interfacial pressure distribution on loosening of bolted joints. Strain, 47 (s2):6578, 2011.

[9] Toru Fukubayashi and Hlsashi Kurosawa. The contact area and pressure distribution pattern of the knee: a study of normal and osteoarthrotic knee joints. Acta Orthopaedica Scandinavica, 51(1-6):871-879, 1980.

\footnotetext{
${ }^{3}$ Since we only measure the change of the contact area and the contact pressure in the interface and not the additional tensile stress in the bolts, this creates the impression of an unloading of the interface at larger response amplitudes.
} 
[10] F Dörner, Ch Körblein, and Ch Schindler. On the accuracy of the pressure measurement film in hertzian contact situations similar to wheel-rail contact applications. Wear, 317(1):241-245, 2014.

[11] Benjamin J Fregly and W Gregory Sawyer. Estimation of discretization errors in contact pressure measurements. Journal of biomechanics, 36(4):609-613, 2003.

[12] Elizabeth I Drewniak, Joseph J Crisco, David B Spenciner, and Braden C Fleming. Accuracy of circular contact area measurements with thin-film pressure sensors. Journal of biomechanics, 40(11):2569-2572, 2007.

[13] Matthew RW Brake. The Mechanics of Jointed Structures: Recent Research and Open Challenges for Developing Predictive Models for Structural Dynamics. Springer, 2017.

[14] Tilàn Dossogne, TW Jerome, DPT Lancereau, Scott Alan Smith, MRW Brake, BR Pacini, Pascal Reuss, and CW Schwingshackl. Experimental assessment of the influence of interface geometries on structural dynamic response. In Dynamics of Coupled Structures, Volume 4, pages 255-261. Springer, 2017.

[15] S. Smith, J. C. Bilbao-Ludena, S. Catalfamo, M. R. W. Brake, P. Reuß, and C. W. Schwingshackl. The effects of boundary conditions, measurement techniques, and excitation type on measurements of the properties of mechanical joints. In Nonlinear Dynamics, Volume 1: Proceedings of the 33rd IMAC, A Conference and Exposition on Structural Dynamics, 2015, pages 415-431. Springer International Publishing, 2016.

[16] S. Catalfamo, S. A. Smith, F. Morlock, M. R. W. Brake, P. Reuß, C. W. Schwingshackl, and W. D. Zhu. Effects of experimental methods on the measurements of a nonlinear structure. In Dynamics of Coupled Structures, Volume 4: Proceedings of the 34th IMAC, A Conference and Exposition on Structural Dynamics 2016, pages 491-500. Springer International Publishing, 2016.

[17] Tekscan Inc. Datasheet pressure mapping sensor 5101, 2017. [Online; accessed 16-October-2017].

[18] Gaetan Kerschen, Keith Worden, Alexander F Vakakis, and Jean-Claude Golinval. Past, present and future of nonlinear system identification in structural dynamics. Mechanical systems and signal processing, 20(3):505-592, 2006.

[19] Brandon J Deaner, Matthew S Allen, Michael J Starr, Daniel J Segalman, and Hartono Sumali. Application of viscous and iwan modal damping models to experimental measurements from bolted structures. Journal of Vibration and Acoustics, 137(2):021012, 2015.

[20] Verein Deutscher Ingenieure. VDI 2230 Part 1: Systematic calculation of high duty bolted joints: Joints with one cylindrical bolt. VDI-guideline. VDI-Gesellschaft Entwicklung Konstruktion Vertrieb, 2003. 